\title{
Coagulopathy related to trauma: Is it time for a goal-directed approach?
}

\author{
Kollengode Ramanathan ${ }^{1}{ }_{F C I C M}$, Bingwen Eugene $\underline{\text { Fan }}{ }^{2}$ MRCP
}

Trauma represents a leading cause of death globally, and post-traumatic haemorrhage accounts for $40 \%$ of early mortality in spite of recent improvements in modern trauma care. ${ }^{1}$ Post-traumatic haemorrhage occurs primarily from direct injury to blood vessels, leading to exsanguination and hypovolaemic shock depending on the type and calibre of the affected vessels. However, one-third of patients with major trauma develop secondary injury from microvascular haemorrhage due to coagulopathy associated with trauma, the pathogenesis of which is multifactorial. ${ }^{2,3}$ Other terminologies that have been proposed to identify this pathological entity include trauma-induced coagulopathy (TIC), traumaassociated coagulopathy, early coagulopathy of trauma, acute traumatic coagulopathy, acute coagulopathy of trauma shock, and coagulopathy of trauma. ${ }^{1,4}$ It is also established that TIC is a multiphenotypic disease state that incorporates coagulation and inflammation cascades. The condition results in impaired clot formation, breakdown, and overall poor vascular homeostasis, contributing to early transfusion requirements, multiorgan failure and mortality. ${ }^{4}$

In this issue of the Annals, Ho et al. present an updated and comprehensive narrative review of literature on coagulopathy associated with trauma. It covers pathophysiology of the condition and emerging advances in management, including rapid diagnosis and goaldirected therapy. ${ }^{5}$ The review is based on a literature search using robust selection of keywords pertaining to coagulopathy of trauma, updated until October 2020. The researchers have highlighted the burning controversies in the area, including the evidence for viscoelastic haemostatic assay-targeted therapy versus the fixed ratio massive transfusion protocols, and outlined some of the ongoing randomised controlled trials whose results are eagerly awaited. However, there are a few aspects of coagulopathy of trauma not covered within the primary aims of the review article and need special mention. The pathophysiological complexities highlighted in the review are further magnified by the lack of uniform definition of TIC, as well as knowledge gaps in the management of a haemorrhagic trauma patient.

The authors summarised that the pathophysiology of coagulopathy of trauma can be dynamic. ${ }^{5}$ Polytrauma patients manifest diverse phenotypes of coagulopathy ranging from hypo- to hyper-coagulability and may quickly shuffle between phenotypes. ${ }^{6}$ In addition to hyperfibrinolysis and fibrinolysis shutdown, endothelial activation, as well as platelet and fibrinogen dysfunction, also occurs in severely injured patients, worsening the secondary coagulopathy. Nevertheless, the review article does not highlight the challenges pertaining to the definition of coagulopathy of trauma. A recent communication by the International Society on Thrombosis and Haemostasis (ISTH) Scientific and Standardization Committee on fibrinolysis, disseminated intravascular coagulation, and perioperative and critical care thrombosis and haemostasis, was published in 2020. The article highlighted the need for a standard definition of TIC while accepting the limitations on timing of blood sampling, as well as not having a standard laboratory method to diagnose and prognosticate it. ${ }^{4}$ The definition of TIC is incomplete by virtue of the variable magnitude of presentation, the early resuscitative practices during the period of tissue injury and shock, in addition to its complex pathophysiology. The committee stratified clinical presentation of TIC as primary versus secondary, early versus late, enhanced versus inhibited fibrinolysis, and responders versus non-responders, in what can be considered as a step forward towards defining this phenomenon.

TIC comprises complex haemostatic dysfunction with likely multiple interrelated pathways, making standard laboratory tests (SLTs) (e.g. international normalised ratio [INR], prothrombin time [PT] and activated partial thromboplastin time [PTT]) poorly reflective of the underlying pathophysiologic processes. While deranged INR is regarded as a major predictor of death and intensive care unit (ICU) length of stay in patients with severe trauma, coagulopathy of trauma can also

\footnotetext{
'Cardiothoracic Intensive Care Unit, National University Hospital, Singapore

${ }^{2}$ Department of Haematology, Tan Tock Seng Hospital, Singapore

Correspondence: Dr Kollengode Ramanathan, Cardiothoracic Intensive Care Unit, Level 2, Main Building, 1E Kent Ridge Road, National University Heart Centre, National University Hospital, Singapore 119228.

Email: ram_ramanathan@nuhs.edu.sg
} 
occur in patients with normal PT/INR and PTT values. ${ }^{1,4}$ However, the emergence of point-of-care tests such as viscoelastic haemostatic assays (VHAs) provides a global approach to evaluating coagulopathy of trauma as compared to SLTs. While SLTs are universally available, they have a longer turnaround analytic time and are primarily designed to evaluate the short initial phase of secondary haemostasis (thrombin generation and formation of a fibrin clot). They do not reflect platelet function and hyper-fibrinolytic activity well. On the other hand, VHAs are point-of-care tests that can yield quicker results and detect the dynamics, stability and sustainability of clot formation, as well as early fibrinolysis. However, VHAs are still unable to provide a sensitive reflection of impaired platelet function. Platelet function remains poorly monitored during trauma resuscitation and while platelet dysfunction has been associated with worse outcomes in trauma, the mechanisms by which platelet dysfunction contributes to coagulopathy are poorly understood. ${ }^{6}$ Ho et al. have highlighted the results of the recently published Implementing Treatment Algorithms for the Correction of Trauma-Induced Coagulopathy (iTACTIC) multicentre randomised controlled trial, which compared the use of VHA-guided to SLT-guided transfusion in adult trauma patients with haemorrhagic shock. ${ }^{7}$ The trial showed no differences in survival or transfusion requirements if they were randomised to the SLT or the VHA arm, as well as in the secondary outcomes (mortality, ventilator-free days, ICU-free days and hospital length of stay). While the iTACTIC trial showed a significant reduction in the timing of interventions in the VHA group, secondary outcomes and post hoc analyses were not adjusted for multiple comparisons. A stepwise sequential VHA algorithm, similar to the one highlighted by Ho et al., ${ }^{5}$ might have been worthwhile looking into in a trauma resuscitation setting. On the contrary, the only other randomised controlled trial that compared SLTs to VHAs in major trauma showed survival benefits with reduction in transfusion requirements. ${ }^{8}$ While the authors have highlighted the major findings from existing randomised controlled trials, the bottom line remains that there is a paucity of high-quality randomised controlled trials or systematic reviews comparing SLTs to VHAs, for an evidence-based approach on this matter.

Ho et al. have also updated the current evidence on tranexamic acid, massive transfusion protocols (MTP) and goal-directed transfusion in this context. While the results of the randomised controlled trials (Prospective, Observational, Multicenter, Major Trauma Transfusion [PROMMTT] study and the Pragmatic, Randomized
Optimal Platelet and Plasma Ratios [PROPPR] trial) were contrasting in terms of the survival outcomes, it needs to be accepted that a fixed ratio MTP may not be the ideal solution for the complex pathophysiology that coagulopathy of trauma entails. ${ }^{9}$ It needs to be emphasised that goal-directed therapies based on coagulation tests (SLT or VHA) are gaining popularity in trauma resuscitation by virtue of its ability to cut down transfusion requirements, mortality and even healthcare costs. A stepwise sequential VHA algorithm might confer a higher predictive value for transfusion needs during trauma and there is a general trend that major trauma centres are embracing this paradigm shift. The role of early administration of fibrinogen in trauma is not yet clear. The availability of cryoprecipitate or fibrinogen concentrate at short notice might pose a challenge to effective implementation globally. However, we need to await the results of ongoing randomised controlled trials for evaluating the utility of fibrinogen concentrates in early trauma. In addition, although the review highlights early studies assessing the addition of 4-factor prothrombin complex concentrates that show a reversal of coagulopathy of trauma and reduction in transfusion requirements, this needs further evaluation before adoption into clinical practice. ${ }^{10}$

Overall, this narrative review emphasises the fact that coagulopathy of trauma needs early identification and prompt treatment. The authors have diligently compiled an evidence-based approach to the conundrum including a sequential algorithm. The proposed approach would be more meaningful if it is supplemented by a proper consensus definition of TIC among multidisciplinary specialists, similar to sepsis-induced coagulopathy, so that the yardstick to stratify and manage the problem remains universally accepted. ${ }^{4}$ Current evidence supports the idea that TIC can be identified by SLTs or VHAs. The limitation of such tests performed in an ex vivo environment is that they do not adequately reflect the crosstalk between the endothelium, circulating platelets, clotting factors, local tissue and traumarelated inflammatory factors. Goal-directed therapy based on such tests is a step forward in reducing inappropriate transfusions when compared to fixed ratio MTP in this setting. Given that resources might vary across hospitals, a dynamic trauma resuscitation protocol that incorporates early MTP in the first hour and moves quickly on to a goal-directed transfusion strategy might be a more practical approach on the ground, so that resuscitation is not delayed in a massively bleeding trauma patient. Administration of tranexamic acid within 3 hours of trauma should be followed; however, its administration in the prehospital 
setting needs to be looked into. ${ }^{9}$ The use of fibrinogen concentrates in early trauma is an interesting concept while we await robust evidence from the randomised controlled trials. Eventually, once we close the knowledge gaps, the snapshot diagnostic assays and reactive management of coagulopathy of trauma should give way to a multivariate in silico modelling of the complex coagulation milieu. ${ }^{1}$ This new future approach may be augmented by computational models and artificial intelligence that can predict dynamic phenotypes of TIC, allowing for more precise and optimal individualised treatment for trauma patients with bleeding.

\section{REFERENCES}

1. Kornblith LZ, Moore HB, Cohen MJ. Trauma-induced coagulopathy: The past, present, and future. J Thromb Haemost 2019;17:852-62.

2. Frith D, Davenport R, Brohi K. Acute traumatic coagulopathy. Curr Opin Anaesthesiol 2012;25:229-34.

3. Savioli G, Ceresa IF, Caneva L, et al. Trauma-Induced Coagulopathy: Overview of an Emerging Medical Problem from Pathophysiology to Outcomes. Medicines (Basel) 2021;8:16.
4. Moore HB, Gando S, Iba T, et al. Defining trauma-induced coagulopathy with respect to future implications for patient management: Communication from the SSC of the ISTH. J Thromb Haemost 2020;18:740-7.

5. Ho VK, Wong J, Martinez A, et al. Trauma-induced coagulopathy: Mechanisms and clinical management. Ann Acad Med Singap 2022:51:40-48

6. Moore HB, Walsh M, Kwaan HC, et al. The Complexity of Trauma-Induced Coagulopathy. Semin Thromb Hemost 2020; 46:114-5.

7. Baksaas-Aasen K, Gall LS, Stensballe J, et al. Viscoelastic haemostatic assay augmented protocols for major trauma haemorrhage (ITACTIC): a randomized, controlled trial. Intensive Care Med 2021;47:49-59.

8. Gonzalez E, Moore EE, Moore HB, et al. Goal-directed Hemostatic Resuscitation of Trauma-induced Coagulopathy: A Pragmatic Randomized Clinical Trial Comparing a Viscoelastic Assay to Conventional Coagulation Assays. Ann Surg 2016;263:1051-9.

9. Spahn DR, Bouillon B, Cerny V, et al. The European guideline on management of major bleeding and coagulopathy following trauma: fifth edition. Crit Care 2019;23:98.

10. Jehan F, Aziz H, O'Keeffe T, et al. The role of four-factor prothrombin complex concentrate in coagulopathy of trauma: A propensity matched analysis. J Trauma Acute Care Surg 2018; 85:18-24. 